\title{
Enabling photonic technologies for seeing-limited telescopes: fabrication of integrated photonic lanterns on a chip
}

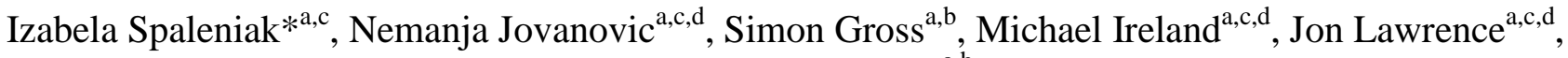 \\ and Michael Withford ${ }^{\mathrm{a}, \mathrm{b}}$ \\ ${ }^{a}$ MQ Photonics Research Centre, Department of Physics and Astronomy, Macquarie University, \\ NSW 2109, Australia \\ ${ }^{\mathrm{b}}$ Centre for Ultrahigh bandwidth Devices for Optical Systems (CUDOS) \\ ${ }^{c}$ Centre for Astronomy, Astrophysics and Astrophotonics, Macquarie University, NSW 2109, \\ Australia \\ ${ }^{\mathrm{d}}$ Australian Astronomical Observatory (AAO), Epping, NSW 2121, Australia \\ *izabela.spaleniak@mq.edu.au
}

\begin{abstract}
In this paper we present theoretical and laboratory results on integrated directly-written photonic lanterns with varying taper lengths. These lanterns convert seeing-limited light into multiple diffraction limited signals, in other words, a multimode signal into multiple single-mode signals. We investigated 19-channel structures which were written within a 30-mm-long glass block and designed to operate at $1550 \mathrm{~nm}$. A single structure consisted of a multimode waveguide which transitioned into an array of single-mode waveguides and then back to a multimode waveguide utilizing cosine taper transitions. Based on simulations we found that transition lengths of $6 \mathrm{~mm}$ were sufficient to obtain throughput at a level of $\sim 95 \%$. Fabricated devices showed losses (coupling and transition losses) at the level of $30 \%$ for injection $F / \#>5$ and taper lengths $>5 \mathrm{~mm}$. We believe that such devices show great promise for future use in astronomy.
\end{abstract}

Keywords: astrophotonics, photonic lanterns, ultrafast direct write, multimode waveguides

\section{INTRODUCTION}

Multimode fibres have been used by astronomers for many years to transport or to reformat light from the telescope focus to instruments placed either at the back end of the telescope or in the Coude room ${ }^{[1]}$. All fibre fed spectrographs used in astronomy so far, work only with multimode (MM) fibre inputs. The large core sizes of MM fibre allow for most of the seeing-limited light to be coupled into the fibre. However, as core size determines the size of a spectrograph in the seeing-limited case; the bigger the core size, the larger the instrument needs to be (in order to maintain a given resolving power). On the other hand, bigger instrument will be more challenging to manufacture and more unstable, because most of current spectrographs employ macroscopic optics. That is why there are definite advantages of using single-mode (SM) fibres, which have much smaller core diameter. What is more, some devices only work with SM inputs ${ }^{[2]}$. A SM input can be obtained from diffraction-limited light, which is usually not the case if we observe through the Earth's atmosphere. Atmospheric turbulences distort the plane wavefronts from the stellar target and changes their phase locally. The size of the co-phased subsections and timescale of their changes is directly related to the wavelength. For visible wavelengths the subsections are much smaller (thus there is more of them) and change much faster than in the near-infrared ${ }^{[3]}$. Over the past decade there has been a rapid development of adaptive optics technologies on large telescopes in order to compensate for the atmosphere and correct the seeing-limited light back into a diffraction-limited image. Despite its efficacy, there are still several major drawbacks which include: high costs, limited corrected field of view and poor operation at shorter wavelengths (due to the required high correction speeds and number of segments required in the visible). Another solution is to convert seeing-limited light into several diffraction-limited beams. This process can be realized by a device that converts a MM fibre core into a series of SM fibre cores which is known as a photonic lantern. Each of the mentioned co-phased wavefront subsections are sampled and guided by the supermodes

Modern Technologies in Space- and Ground-based Telescopes and Instrumentation II,

edited by Ramón Navarro, Colin R. Cunningham, Eric Prieto, Proc. of SPIE Vol. 8450, 845015

(c) 2012 SPIE $\cdot$ CCC code: 0277-786X/12/\$18 - doi: 10.1117/12.925264 
in a MM fibre. Then the supermodes can be efficiently transformed into a series of single modes, if the number of supermodes is equal to the number of individual single modes at the output and if thetransition is adiabatic [ref. Sergio].

The principle of such photonic lanterns was first shown in $2005^{[4]}$ but the first efficient photonic lantern was demonstrated in $2009^{[5]}$. There are two approaches to photonic lantern fabrication. The first method uses a bundle of SM fibres surrounded by a capillary tube which is then tapered down. The region where the fibres have collapsed and moulded together acts as a MM waveguide ${ }^{[5]}$. Up to now $1 \times 7,1 \times 19$ and $1 \times 61$ photonic lanterns (1 MM input to 7, 19, 61 SM outputs, respectively) have been demonstrated ${ }^{[4]-[6]}$. The other method uses the laser direct write technique to inscribe the waveguides into a glass sample with an ultrafast laser. The main advantages of this technique are as follows. First, the device can be miniaturised: instead of a minimum $127 \mu \mathrm{m}$ separation of single-mode fibres, an integrated photonic lantern could have as little as $10 \mu \mathrm{m}$ separation of guides. This means that the integrated device would be at least ten times smaller than the fibre based one. Secondly, the device can be reformatted into arbitrary output (e.g. slit) and finally, in principle it can have an unlimited number of waveguides. The recently demonstrated integrated photonic lantern consisted of $16 \mathrm{SM}$ waveguides, which were brought together from a single square-shaped MM waveguide ${ }^{[7]}$. The losses of that device were estimated to be very low, i.e. $\sim 2 \mathrm{~dB}$.

Although a directly written photonic lantern has been demonstrated with one set of component parameters, there is a large parameter space that can be explored in order to better understand and hence optimise future devices. Indeed with such an investigation it should be possible to fabricate high throughput photonic lanterns, high mode count photonic lanterns and even photonic lanterns operating at visible wavelengths, which is the ultimate aim for this project. There are many variables we are actively changing in order to achieve these goals, including the transition length between multimode and single-mode sections (referred as taper length). In this article we present a summary of the characterization of a prototype device operating at $1550 \mathrm{~nm}$ manufactured with a femtosecond laser and taper transition modelling of other device geometries we are currently exploring.

\section{DESIGN AND MODELLING}

\subsection{Structure design}

The structures were designed to operate at $1550 \mathrm{~nm}$ and support 19 modes. They consisted of multimode - taper - single mode (MM - SM) sections (Figure 1). The MM waveguides were composed of a lattice of 19 SM waveguides arranged in a circular array. The size of a SM waveguide was $\sim 10 \mu \mathrm{m}$ and separation between the waveguides was also $10 \mu \mathrm{m}$, so that the waveguides had no overlap but they were sufficiently close enough to allow cross-couping and hence supermodes to be supported by the structure. This design also ensured that the glass was not modified more than once throughout the entire block.

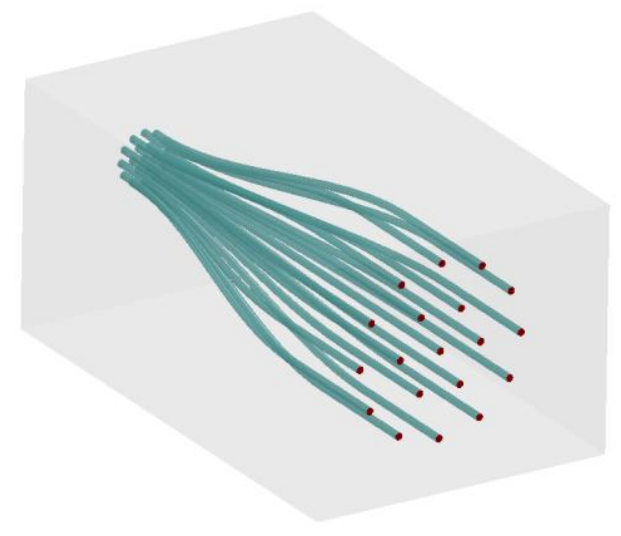

Figure 1.CAD model of a photonic lantern used in modeling. 


\subsection{Taper transition}

We considered three types of transitions: linear, cosine and raised sine as well as the length of the taper transition (Figure 2). The transition length was varied between $1-24 \mathrm{~mm}$.

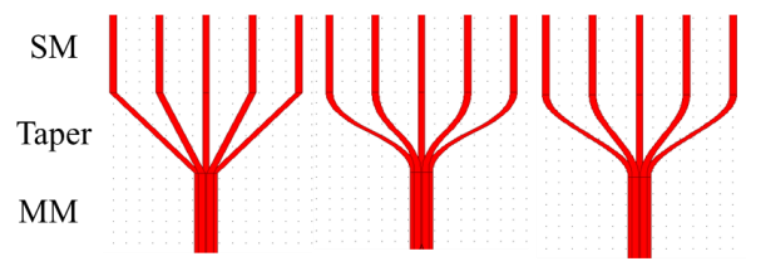

a) Linear b) Raised Sine c) Cosine

Figure 2. Three taper transition types used in modeling (note: picture is not in scale).

\subsection{Modeling results}

Photonic lanterns were modelled and simulated using a commercial implementation of the beam propagation algorithm BeamPROP package in $\mathrm{R}$-soft ${ }^{[8]}$.A multimode injection field with $\mathrm{f} / 7$ focal ratio was injected into the structures. Transmitted power was measured by a total power monitor. In order to ensure that only power guided in the structure was measured, a very long SM section was set at the end of the transition, so that the cladding modes would reach the boundaries and terminate (Figure 3).

Figure 4 shows transmission results for MM - SM photonic lantern transition. It can be seen that the transmission similar across all transition types considered, although it is slightly higher for the cosine transition type. For taper lengths between $1 \mathrm{~mm}$ and $5 \mathrm{~mm}$, losses strongly depend on the taper length. For taper lengths $>6 \mathrm{~mm}$ the transmission reaches $\sim 95 \%$ and flattens off.
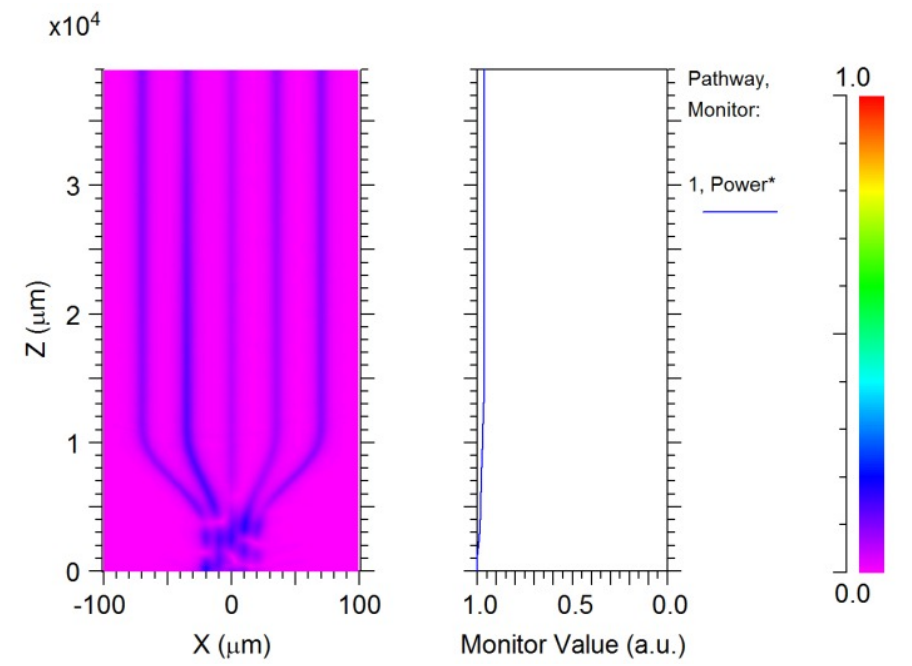

Figure 3. BeamPROP example simulation for $10 \mathrm{~mm}$ raised sine taper transition. Left side: shows propagation of the electromagnetic field across the structure; right side: total power monitor value along the structure. 


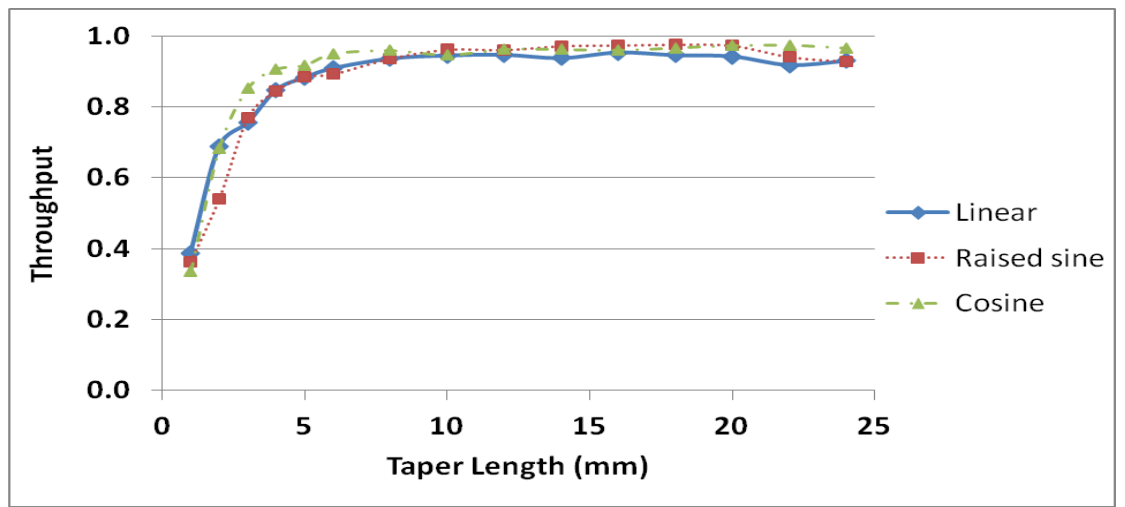

Figure 4. Simulated throughput as a function of taper transition length for three different transition types: linear, raised sine and cosine.

\section{FABRICATION OF THE PHOTONIC LANTERNS}

\subsection{Waveguide writing parameters}

The photonic lanterns were inscribed by means of the direct write technique. In this technique a laser beam with ultrashort pulses is focused into a glass sample and locally modifies the refractive index of the glass. By moving the sample in the XYZ direction, waveguides are written (Figure 5). The system used to inscribe photonic lanterns presented in this paper consists of the following elements: femtosecond laser operating at $800 \mathrm{~nm}$ with pulse duration $<50 \mathrm{fs}$ and repetition rate of $\sim 5.1 \mathrm{MHz}$; 100x oil immersion objective lens with numerical aperture $(N A)$ of 1.25; Aerotech, airbearing translation stages. The sample was a 30-mm-long boro-aluminosilicate (Corning Eagle2000) glass sample. The structures were written with $35 \mathrm{~nJ}$ pulse energy at $750 \mathrm{~mm} /$ minute translation velocity. These parameters provide a SM waveguide at $1550 \mathrm{~nm}$. As mentioned the size of such waveguides was approximately $10 \mu \mathrm{m}$ and separation between the waveguides in the MM section was also $10 \mu \mathrm{m}$. Waveguides were written with a cosine type taper transition, which showed the best throughput from the modelling section (ref. Section 2.3). For characterization simplicity photonic lanterns were composed of MM - Taper - SM - Taper - MM sections with the following taper lengths: 1, 3, 5, 6, 8, 10 and $12 \mathrm{~mm}$. After the inscription, the chip was ground and polished to reveal the waveguide ends. $0.75 \mathrm{~mm}$ was removed off each end, which resulted in a $28.5 \mathrm{~mm}$ final chip length.

\subsection{Waveguide writing process}

The process allowing for a waveguide inscription is called a multiphoton absorption. Dielectric materials, such as boroaluminosilicate glass Eagle2000 have a large band gap, so they are completely transparent at $800 \mathrm{~nm}$. However, if the incident electric field strength is high enough (approximately equal to the electric field that binds the valence electrons) electrons can be excited, thermalised with the ions and the material structure can be changed permanently (which brings about a refractive index modification). This effect can happen if several photons are incident on an electron at the same time and cause a mulitphoton absorption ${ }^{[9]}$. It can be achieved by tight focusing of the laser light with high $N A$ objective lens. The modified feature size depends on the objective $N A$, pulse energy and time of interaction between the laser light and the substrate. The interaction time can be controlled by the translation velocity of the stages in our case.

The system used to write waveguides presented in this paper operates in the high repetition rate regime known as the cumulative-heating regime. This means that the glass does not fully cool down after interaction with an initial pulse before the next pulse arrives to modify the glass further. As a result the refractive index modification has a complex profile but the high repetition rate allows for very fast waveguide's inscription ( $\sim 3 \mathrm{~s}$ for a single waveguide). 


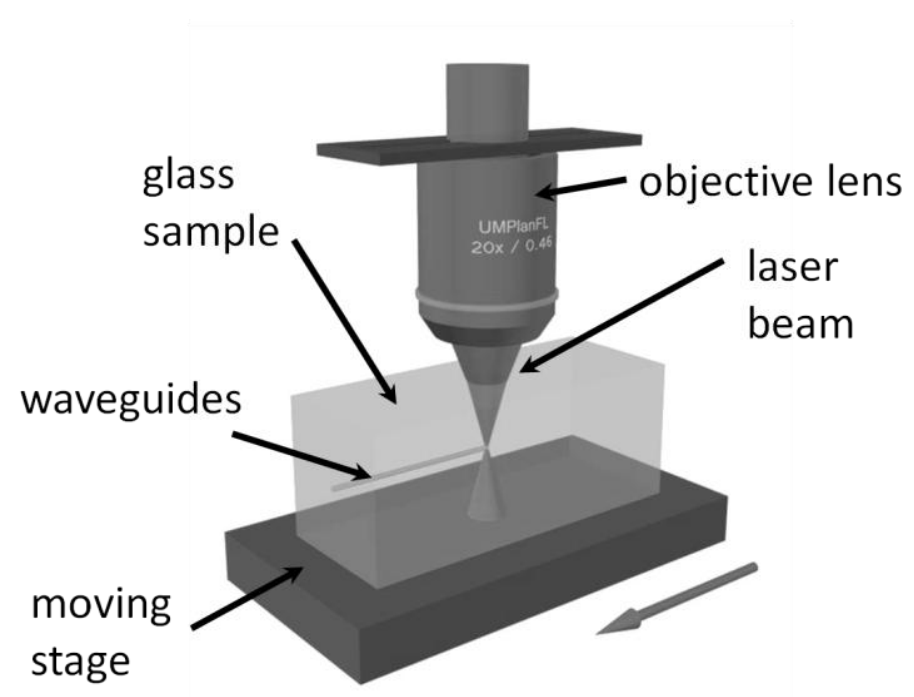

Figure 5. Schematic diagram of direct write setup. Ultrashort laser pulse is focused by an objective lens onto a glass sample. As a result a local refractive index change is created. Stage moves the sample, so that a $3 \mathrm{D}$ waveguide is inscribed.

\section{CHARACTERISATION AND RESULTS}

\subsection{Fabricated structures}

For characterization simplicity the photonic lanterns were composed of MM - Taper - SM - Taper - MM sections (Figure $6 b)$ ). Input and output MM waveguides fill a $50 \mu \mathrm{m}$ diameter spot (Figure $6 \mathrm{a})$ ).
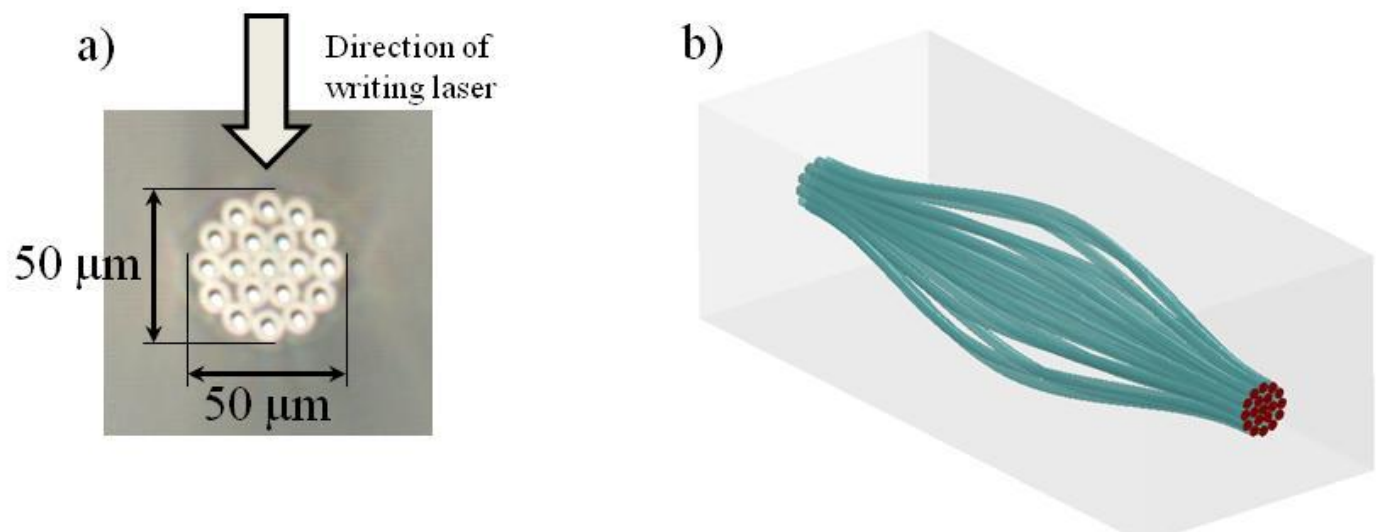

Figure 6. a) Microscopic image of the MM input of the photonic lantern and b) CAD drawing of the whole structure.

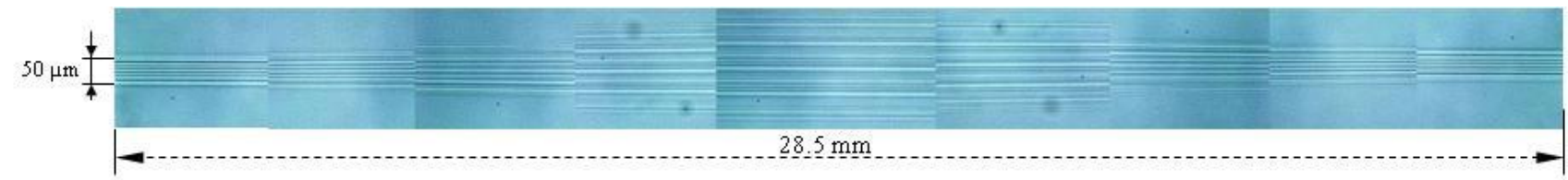

Figure 7. Microscopic image of several segments of the photonic lantern taken with a differential interference contrast microscope in transmission. Parts of the image were removed in order to fit the aspect ratio. 


\subsection{Throughput measurement setup}

The throughput of the structures was measured with a setup shown in Figure 8. Laser light from the SuperK supercontinuum laser source (NKT Photonics) was coupled directly into a SM fibre. The SuperK emits light between $500 \mathrm{~nm}$ and $2400 \mathrm{~nm}$, so in order to select certain wavelength, a $1500 \pm 2.4 \mathrm{~nm}$ bandpass filter was inserted into a collimated beam. The filtered light was then coupled into a $50 \mu \mathrm{m}$ core diameter, $0.22 \mathrm{NA}, 3-\mathrm{m}$-long MM fibre. The output of the fibre was collimated by a $30 \mathrm{~mm}$ focal length achromat and focused again by a $25 \mathrm{~mm}$ focal length achromat. In order to control the focal ratio/numerical aperture $(F / \# / N A)$ of the beam injected to the photonic lanterns, an iris was placed in the collimated beam. An iris, collimating and injection optics were all mounted on a 6-axis translation stage, which allowed for fine adjustment of the coupling into the photonic lanterns. A $100 \mu \mathrm{m}$ core diameter, 0.22 NA MM fibre was used for collecting the light at the output of photonic lanterns. Both sample and coupling fibre were placed on translation stages which allowed for fine position adjustment. The end of the coupling fibre was imaged onto a germanium photodetector, which was monitored by a power meter.

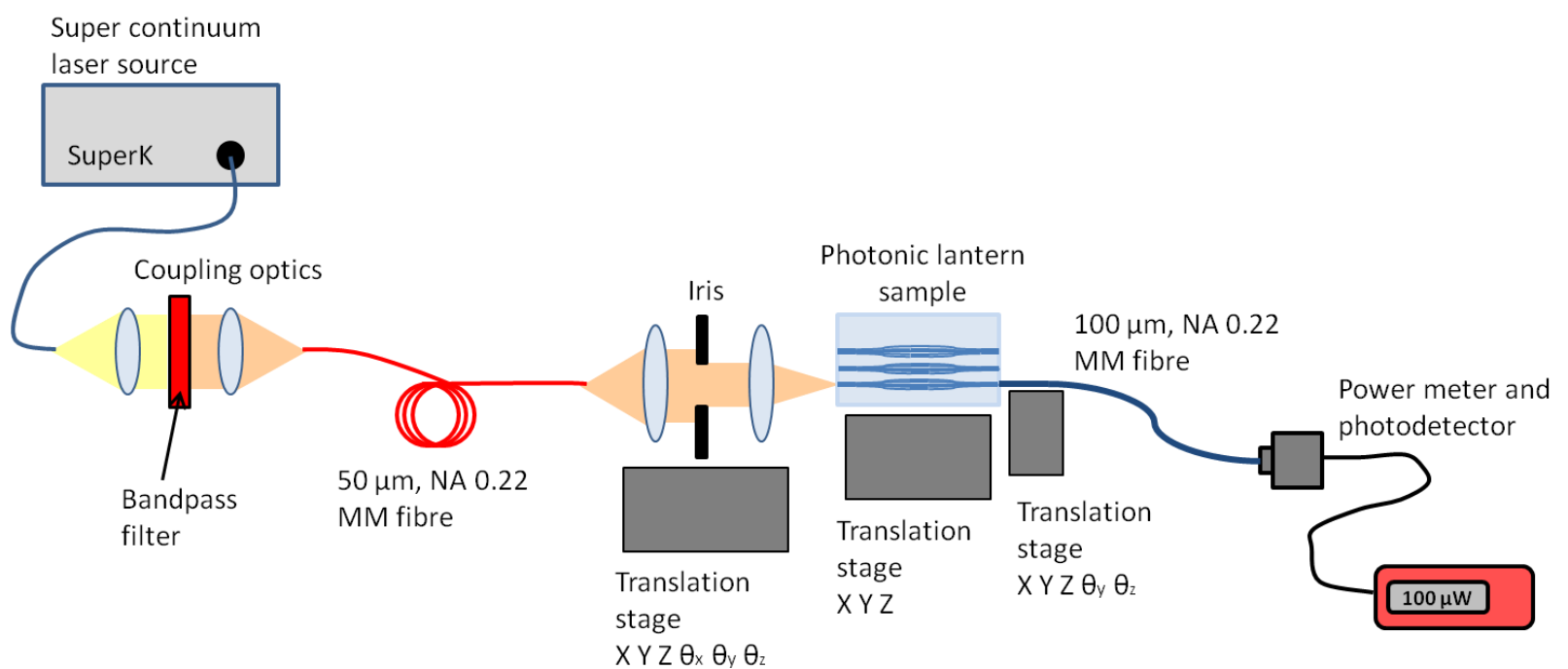

Figure 8. Schematic of the experimental setup used to probe the losses of the photonic lanterns.

\subsection{Throughput results and discussion}

Figure 9 summarizes normalized throughput measurement results for seven photonic lanterns with different taper transition lengths of $1,3,5,6,8,10$ and $12 \mathrm{~mm}$. Each line denotes different injection $F / \#$. Throughput values were corrected for absorption losses (absorption coefficient for Eagle2000 at $1500 \mathrm{~nm}$ is $0.0075 \mathrm{~mm}^{-1}$ ), so the presented values include coupling losses and transition losses (losses due to taper length). As expected from the modelling, throughput increases rapidly between a taper length of 1 and $5 \mathrm{~mm}$. In addition, the throughput increases with increasing $F / \#$ of injection (decreasing $N A$ ). This is because the cut-off for such MM waveguides is $\sim F / \# 9-10^{[9]}$, so low $F / \#$ s are above the acceptance angle of the waveguides. These preliminary results suffer strongly from instability of the SuperK source, giving errors of the order of $20 \%$. Other errors may arise from imperfections in fabricated waveguides. Nevertheless, a throughput at the order of $70 \%$ (including coupling losses) for a very short $(\sim 5 \mathrm{~mm})$ transition length indicates that it will be possible to use short glass samples in future.

This is the first study investigating different taper transition types and lengths in directly laser written photonic lanterns. It is especially important for future multiple channel photonic lanterns as well as fan-out (slit-like end) devices. 


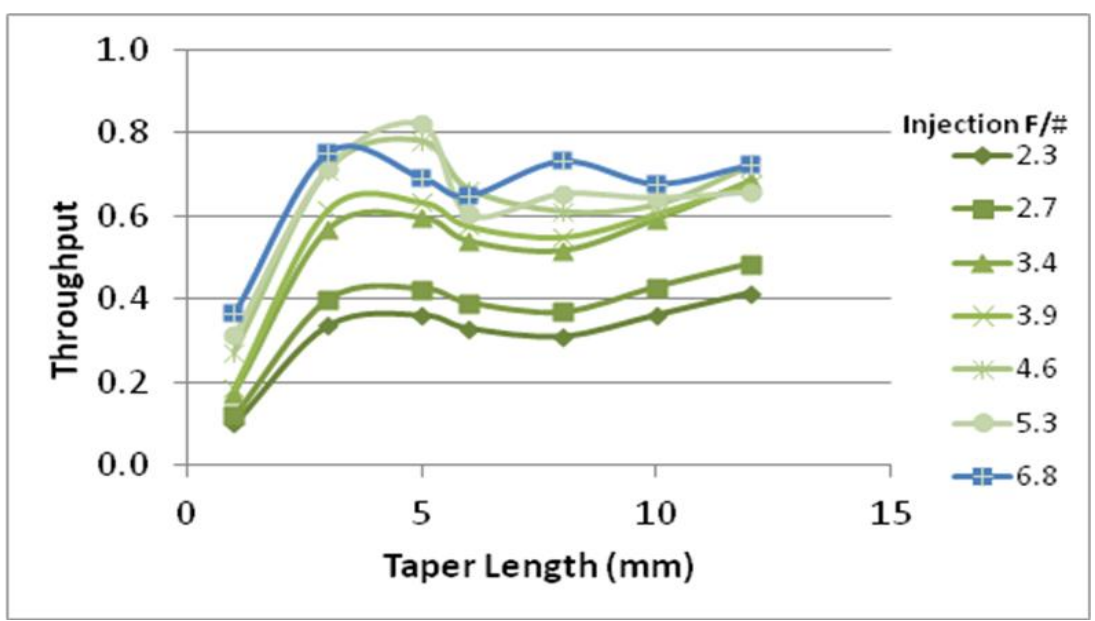

Figure 9. Photonic lantern throughput as a function of taper length for various injection $F / \#$ probed at $1500 \mathrm{~nm}$. A correction has been made for the glass transmission, but no correction has been made for coupling losses. The maximum throughput excluding coupling losses in other experiments ${ }^{10}$ is consistent with $100 \%$ (with 5\% errors).

\section{CONCLUSIONS}

In this publication we reported on the use of the direct write technique to fabricate infrared photonic lanterns on a chip. We investigated different transition types and their lengths in order to maximize the throughput. Modeling showed that a very good transmission (>95\%) can be obtained for taper lengths more than $8 \mathrm{~mm}$ long. Experimental results followed this trend and resulted in maximal transmission at the order of $70 \%$ (which includes also coupling losses). Although these are preliminary results and there is still plenty of room to optimize photonic lanterns on chip, we can conclude that they give are promising for miniaturizing seeing-limited to diffraction-limited converting devices.

\section{ACKNOWLEDGEMENTS}

This research was conducted with the Australian Research Council Centre of Excellence for Ultrahigh Bandwidth Devices for Optical Systems (project number CE110001018) and the assistance of the LIEF and Discovery Project programs.

\section{REFERENCES}

[1] Bland-Hawthorn, J. and Kern, P., "Astrophotonics: a new era for astronomical instruments,” Opt. Express 17(3), 1880-1884 (2009).

[2] Cvetojevic, N., et al., "Characterization and on-sky demonstration of an integrated photonic spectrograph for astronomy," Opt. Express 17(21), 8643-18650 (2009).

[3] Duchêne, G., "High-angular resolution imaging of disks and planet," New Astronomy Reviews 52(2-5), 117-144 (2008).

[4] Leon-Saval, S.G., A. Argyros, and J. Bland-Hawthorn, "Photonic lanterns: a study of light propagation in multimode to single-mode converters," Opt. Express 18(8), 8430-8439 (2010).

[5] Noordegraaf, D., et al., "Efficient multi-mode to single-mode coupling in a photonic lanter," Opt. Express 17(3), 1988-1994 (2009).

[6] Noordegraaf, D., et al., "Multi-mode to single-mode conversion in a 61 port Photonic Lantern," Opt. Express 18(5), 4673-4678 (2010). 
[7] Thomson, R.R., et al., "Ultrafast laser inscription of an integrated photonic lantern,” Opt. Express 19(6), 5698-5705 (2011).

[8] RSoft BeamPROP, http://www.rsoftdesign.com/ (June 2012).

[9] Gattass, R.R. and Mazur, E., "Femtosecond laser micromachining in transparent materials," Nat Photon 2(4), 219225 (2008).

[10] Jovanovic N., et al., "Integrated photonic building blocks for next-generation astronomical instrumentation: the multimode waveguide," Submitted to Optics Express (2012). 